\title{
Long-Acting Injectable GLP-I Receptor Agonists for the Treatment of Adults with Type 2 Diabetes: Perspectives from Clinical Practice
}

This article was published in the following Dove Press journal:

Diabetes, Metabolic Syndrome and Obesity: Targets and Therapy

\section{Mario Luca Morieri 1 \\ Angelo Avogaro (D) \\ Gian Paolo Fadini}

Department of Medicine, University of Padova, Padova 35/28, Italy
Correspondence: Mario Luca Morieri Department of Medicine, University of Padova, Via Giustiniani 2, Padova 35/28, Italy

Tel +390498217094

Email morieri.mL@gmail.com

\begin{abstract}
Randomized controlled trials (RCTs) have consistently shown glycemic and extra-glycemic benefits of long-acting injectable glucagon-like-peptide-1 receptor agonists (GLP-1RAs, liraglutide, albiglutide, exenatide once-weekly, dulaglutide, and semaglutide) in terms of reduction in the rates of cardiovascular events and mortality among patients with type 2 diabetes. Recently, the analyses of large datasets collecting routinely-accumulated data from clinical practice (ie, real-world studies, RWS) have provided new opportunities to complement the information obtained from RCTs. In this narrative review, we addressed clinically relevant questions that might be answered by well-conducted RWS: are subjects treated with GLP-1RAs in the "real-world" similar to those included in RCTs? Is the performance of GLP-1RA observed in the RWS (effectiveness) similar to that described in RCTs (efficacy)? Is the effectiveness similar in population of patients generally underrepresented in RCTs? Are the cardiovascular benefits of GLP-1RAs confirmed in RWS? We also describe a few comparisons currently un-explored by specific RCTs, such as direct comparison between different administration strategies (eg, fixed- versus flexiblecombination with basal-insulin) or between GLP-1RAs versus dipeptidyl-peptidase-4 inhibitor (DDP4i) or versus sodium/glucose cotransporter-2 inhibitors (SGLT-2i) on hard cardiorenal outcomes. Altogether, RWS provide highly informative information on treatment with GLP-1RAs. On the one side, RWS showed different clinical characteristics between subjects enrolled in RCTs versus those attending real-world clinics and receiving a GLP-1RA. On the other hand, RWS showed that GLP-1RA effectiveness is overall consistent in subgroups of patients less represented in RCTs. In addition, RWS allowed the identification of modifiable factors (eg, titration or adherence) that might guide physicians towards better GLP-1RAs use. Finally, multiple RWS reported better cardio-renal outcomes with GLP-1RAs than with DPP$4 \mathrm{i}$, while initial findings from RWS described a weaker cardiovascular protection compared to SGLT-2i. Therefore, there is the need for further RWS and RCTs comparing these different classes of glucose lowering medications.
\end{abstract}

Keywords: observational studies, real-world evidence, effectiveness, cardiovascular prevention, head-to-head comparisons, innovative

\section{Introduction}

Glucagon-like-peptide-1 receptors agonists (GLP-1RAs) are relatively novel drugs for the treatment of type 2 diabetes (T2D) that stimulate the incretin hormone GLP1. ${ }^{1}$ GLP-1RAs can be distinguished, according to their half-life and time-action profile, as short-acting (exenatide and lixisenatide) or long-acting (exenatide onceweekly, liraglutide, albiglutide, dulaglutide and semaglutide). 
The treatment with GLP-1RA provides beneficial effects that goes beyond the improvement of glucose homeostasis (also known as glycemic-effect). ${ }^{1,2}$ Indeed, as described in detail in previous reviews, ${ }^{1,3-5}$ several extra-glycemic effects of GLP-1RAs have been identified. Among these, GLP-1RAs are able to reduce body weight (delaying gastric emptying and increasing satiety) and to reduce blood pressure (through reduction in body weight, but also trough increasing natriuresis and inducing vasodilation), as well as to improve microcirculation perfusion, endothelial function and lipid profile (reducing low-dense-lipoprotein and triglyceride-rich lipoproteins concentrations). ${ }^{1,3-5}$ Most importantly, four different long-acting GLP-1RAs (liraglutide, albiglutide, semaglutide and dulaglutide) have been found to the reduce risk of future major adverse cardiovascular events (MACE, including cardiovascular death, non-fatal myocardial infarction and stroke) in patients with T2D, with the other long-acting GLP-1RAs, exenatide onceweekly (exeOW) showing a trend towards reduction in MACE combined with a nominally significant reduction in overall mortality. ${ }^{6-9}$ When combined together in a metaanalyses, cardiovascular outcome trials on GLP-1RAs have showed efficacy in reducing occurrence of cardiovascular and renal outcomes (progression of albuminuria), and of overall mortality. ${ }^{10}$

The outstanding results on cardio-renal hard outcomes have been found within verywell conducted randomized controlled trials (RCTs), where the rigorous methodology and controlled clinical setting have allowed obtaining reliable results, with high internal validity, free of possible confounding effects (from either measured or unmeasured factors) thanks to the randomized allocation of subjects to intervention or placebo. However, application of such a rigorous experimental setting can lead to stunning differences from the real-world routine clinical practice setting.

For these reasons, when researchers and physicians try to translate results from RCTs to their clinical practice, as usually is done in guidelines, several questions arise. For instance, how representative are RCTs of the population with $\mathrm{T} 2 \mathrm{D}$ attending the real-world clinics? Are the clinical characteristics of subjects treated with these drugs similar to those of subjects enrolled in RCTs? If the population is different, are the results of RCTs still valid? If the population treated has similar clinical characteristics, but the settings are different (eg, frequency of follow-up visits, frequency physician/nurse contacts with patients, and adherence) are the results of RCTs transferrable to the real-world setting? Are there other factors that might influence the effectiveness of GLP-1RAs in the real-world clinical practice, including prescription patterns, eg, therapeutic inertia?

For all these reasons, as depicted in Figure 1, real-world studies (RWS) can provide useful and relevant information on the use of GLP-1RAs beyond those observed in RCTs. ${ }^{11,12}$ Moreover, RWS provide opportunities to explore comparisons not tested in RCTs. In this review we addressed such relevant questions and highlight perspectives derived from RWS on long-acting GLP-1RAs.

\section{Literature Search}

To answer the questions addressed in this narrative review, we have included articles in English, published in PubMed or Web Of Science up to August 2020. The following keywords have been searched: "GLP-1RAs", "observational

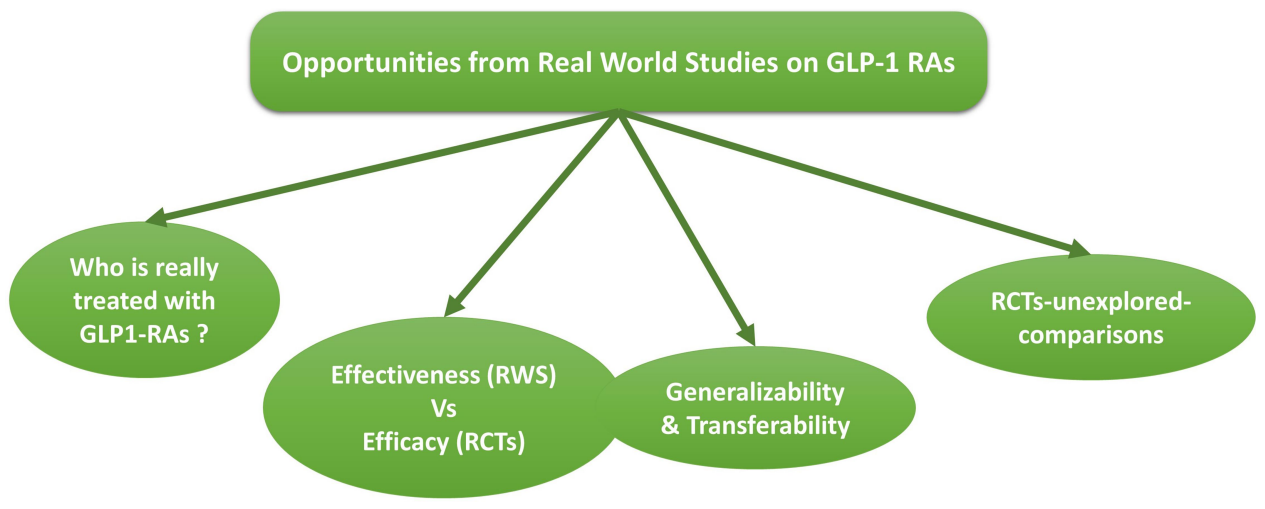

Figure I Opportunities from real-world studies (RWS) to implement notions from randomized controlled trials (RCTs). The figures describes some of those research questions that can be addressed with RWS, eg, the evaluation of whether subjects treated with GLPI-RAs have clinical characteristics similar to those enrolled in RCTs, or whether the benefit of GLP-IRAs described in RCTs are confirmed in RWS and in different populations (generalizability). Finally, RWS provide the opportunity to explore some head-to-head comparisons not yet tested in RCTs. 
studies", "real-world studies" or "real-world evidence", "exenatide once-weekly", "liraglutide", "albiglutide", "dulaglutide" and "semaglutide". We have included original articles, reviews or meta-analyses, and abstracts to the American Diabetes Association (ADA) or European Association for the Study of Diabetes (EASD) meeting.

\section{From Experimental Randomized Controlled Trial to Real-World Clinical Practice}

One of the major reasons for which RWS can provide several pieces of information that are complementary to those retrieved from RCTs resides in the differences in the clinical characteristics of patients enrolled in RCTs as compared to those of patients that physicians encounter in her/ his routine clinical activity and decide to prescribe with a given medication. This general problem is found in several fields of medicine, ${ }^{13-15}$ including the specific field of clinical trial on GLP-1RAs in patients with diabetes. In a recent multicenter study conducted on over 280,000 subjects with T2D treated in outpatients diabetes clinic in Italy, our group showed that, at most, one-third of these "real-world" patients would be included in each specific cardiovascular outcome trial (CVOT) on GLP-1RAs according to inclusion/exclusion (I/E) criteria (Figure 2). ${ }^{16}$ Moreover, more than $50 \%$ of subjects attending these clinics, representative of all diabetes subjects in Italy, would not be included in any of these trials. Similar results have also been reported from analyses of patients with T2D treated in the Unites States. ${ }^{17}$ In addition, after applying sophisticated approaches of data analyses to select a group of subjects with CVOT-like clinical characteristics (ie, with average clinical characteristics similar to CVOT), we found that the proportion of subjects selected from real-world clinical practice would be at most $8 \%{ }^{16}$

\section{Perspective}

Conducting RWS is highly important to understand whether subjects treated with these drugs in clinical practice have characteristics similar to those enrolled in RCTs and, if not (as it is), whether the efficacy found in RCTs is confirmed in clinical practice (ie, generalizability of effectiveness). Moreover, these observations support the need to improve external validity and generalizability of RCTs,

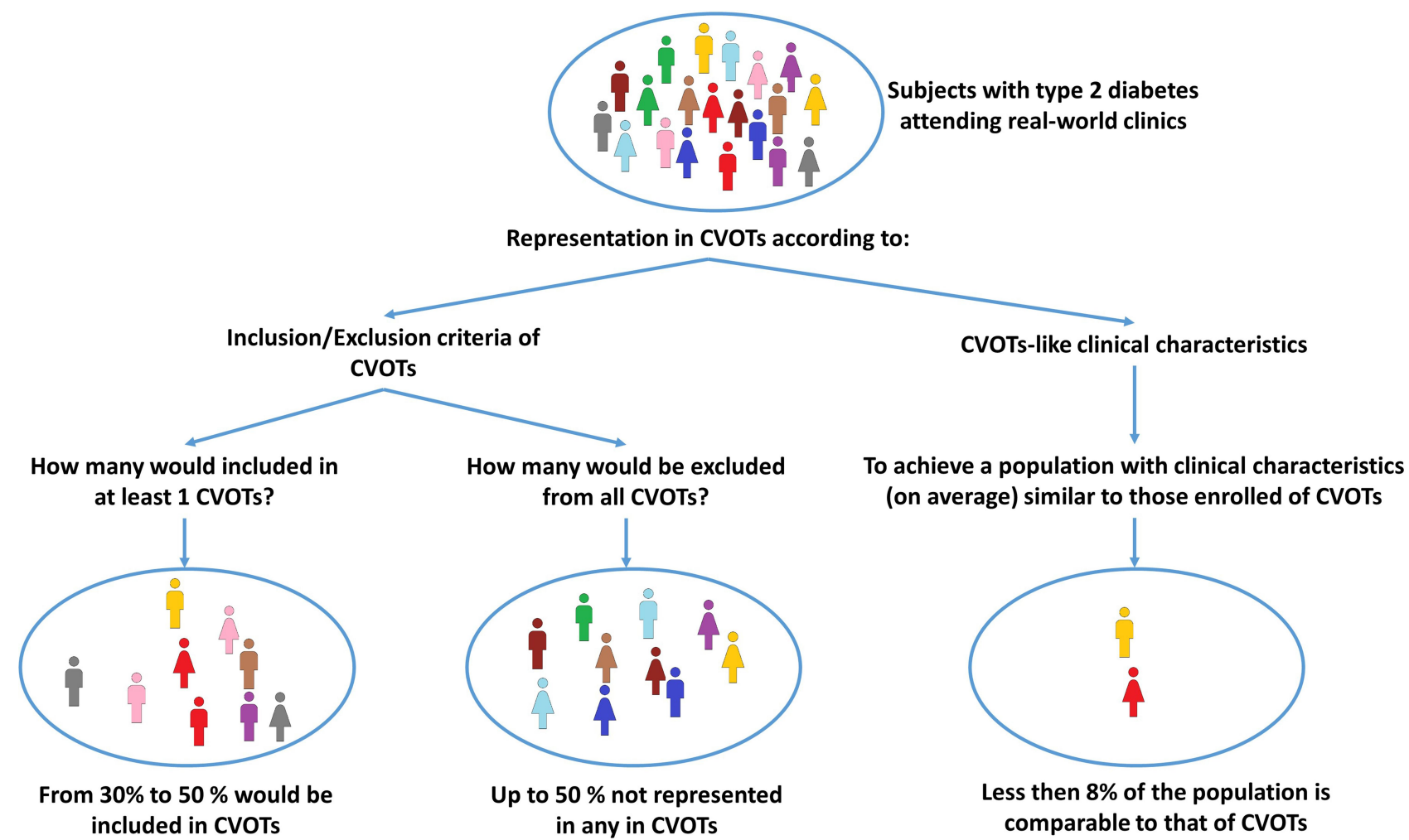

Figure 2 Lack of adequate representation in cardiovascular outcome trials (CVOTs) of subjects with type 2 diabetes attending real-world clinics. The figure describes the proportion of subjects with type 2 diabetes attending "real-world" diabetes centers that can be considered to be adequately represented in CVOTs according to two possible evaluations: the first and second from the left is according to Inclusion/Exclusion (I/E) of the trials, thethird fromthe left is according to the goal of selecting a population of subjects with clinical characteristics similar on average to those population enrolled in CVOTs. 
for instance avoiding unduly restrictive inclusion and exclusion criteria and using electronic health care record systems to enhanced recruitment of subjects and derive broader and more generalizable results. ${ }^{18}$

\section{Description of Subjects Being Treated with Long-Acting GLP-I RAs in the Real-World Setting}

The efficacy of GLP-1RAs on glycemic and extraglycemic targets observed in RCTs, and most importantly on reduction of cardiovascular events and overall mortality, have led scientific societies to modify clinical guidelines with recommendations towards an early use of GLP1RA as second-line treatments, as an alternative or in combination with sodium/glucose cotransporter 2-inhibitors (SGLT-2i). ${ }^{19}$ This has been paralleled by an increase in prescription of these drugs over the last 10 years. ${ }^{20}$ However, guideline recommendations are often not readily implemented into routine clinical practice ${ }^{21}$ and therefore patients treated with these new classes of drugs might be different from those enrolled in CVOTs and also from those guidelines would suggest to treat.

RWS in this context are essential to provide a picture of the clinical characteristics of patients treated with GLP1RAs in clinical practice. For instance, a recent study has described the characteristics of subjects initiating GLP1RAs between 2014 and 2015 in Catalonia (Spain). ${ }^{22}$ From the analyses of general practitioners health care data covering $74 \%$ of the Catalonia regions, the authors found that GLP-1RAs were generally prescribed to middle-aged person, with diabetes lasting for around 10 years, with poor glycemic control (HbA1c $>8 \%$ ), overall obese and, in around $15 \%$ of cases, with pre-existing ischemic heart disease. ${ }^{22}$ Similar clinical characteristics have been reported in a multicenter-study from north-east Italy regions, collecting electronic health data of 83,116 subjects with T2D. ${ }^{23}$ Moreover, in the study conducted by our group, the prescription patterns of GLP-1RAs over calendar years (between 2010 and 2018) was consistent with a higher use over time among subjects with more advanced disease stage. This was paralleled by an increase in prescriptions of GLP-1RAs among subjects on secondary prevention (that doubled from $8 \%$ in 2010 to $16 \%$ in 2018), and as fixed or flexible combination with insulin.

Both these studies, from Italy and Spain, showed also how the characteristics of patients treated with GLP-1RAs were markedly influenced by local reimbursement criteria (eg, specific $\mathrm{HbA} 1 \mathrm{c}$ or BMI, criteria, etc.) more than changes in guideline recommendations. ${ }^{22,23}$

A similar Danish study analysed the trend of prescription of GLP-1RAs between 2014 and 2017. ${ }^{24}$ The treated subjects had similar age and $\mathrm{HbAlc}$ of that reported in Italy, although had a shorter duration of diabetes but with a higher cardiovascular burden (around 30\% had established cardiovascular disease). Contrary to our report, there was no increase over years towards more prescription among those with established cardiovascular disease, and specifically they found no influences in prescription approaches after publication of ground-breaking CVOTs, such as the LEADER (Liraglutide Effect and Action in Diabetes: Evaluation of Cardiovascular Outcome Results) study.

\section{Perspective}

Altogether, these data suggest that GLP-1RAs are generally used more frequently in advanced disease stages and highlight how country- or region-specific RWS can carry useful clinical information on subjects actually treated with GLP-1RAs in clinical practice. This allows the identification of guideline recommendations that might be overlooked and that instead need to be reinforced (or modified) by national and international societies. In addition, RWS can highlight whether reimbursement criteria are limiting the opportunity to provide the best (and hopefully cost-effective) treatments for patients.

\section{Effectiveness vs Efficacy}

As outlined in the previous paragraphs, RCTs and real-world clinical practice differ for both the environment (highly controlled in RCTs vs highly variable in clinical practice) and for the population (highly selected in RCTs vs unselected in routine clinical practice). Therefore, RCTs can lack external validity and may not be generalizable to the population actually treated in the real-world setting where the characteristics might be diverse. For these reasons, the performance of an intervention in RCTs or RWS is generally described using two different terms, efficacy and effectiveness, respectively. ${ }^{25}$ Efficacy refers to the performance of the intervention in the ideal and experimental setting of trials and addresses the question of whether an intervention can work. Effectiveness describes the performance of the same intervention in "real-world" circumstances and addresses the question of whether it actually works in everyday conditions.

Although RWS are not expected to substitute for RCTs in the assessment of performance of clinical 
interventions, ${ }^{11}$ they can provide highly useful opportunities to investigate areas not covered by RCTs. For example, the majority of RCTs are placebo-controlled and only a few RCTs are designed to provide head-to-head comparison between different active compounds, an aspect that is highly relevant from a clinical standpoint, since in the "real-world" physicians more often choose between one drug and another as compared to one drug or nothing. Another opportunity provided by RWS is to investigate the performance of interventions in populations that are generally under-represented in clinical trials (eg, in the elderly, in race/ethnic minorities, or in subjects with chronic kidney disease and other comorbidities).

\section{Glycemic and Extra-Glycemic Effectiveness \\ Intraclass Comparisons Among GLP-IRAs}

As far as GLP-1RA treatments are concerned, few RCTs have tested different compounds in head-to-head comparisons, ${ }^{26-31}$ and often these types of comparison are described by means of network-meta-analyses (ie, using a common comparator as reference, although the studies are conducted in different populations) ${ }^{32-34}$ or as metaregression. ${ }^{35,36}$ However, as described in Table 1, results of RCTs have not always been confirmed in observational studies derived from "real-world" clinical practice. These RWS, that are not intended to question the efficacy of drugs as determined in RCTs, have however allowed highlighting relevant pieces of information with implications from clinical points of view. For instance, while RCTs showed that dulaglutide $1.5 \mathrm{mg}$ and liraglutide $1.8 \mathrm{mg}$ are equivalent in terms of $\mathrm{HbA} 1 \mathrm{c}$ reduction (as described in the AWARD-6 trial), ${ }^{29}$ RWS found that, after adjusting for several confounders, subjects treated with dulaglutide achieve better
HbA1c control compared to liraglutide users. ${ }^{37}$ These differences were explained with the lack of adequate uptitration of liraglutide in real-world setting, where less than $20 \%$ of subjects achieved the full $1.8 \mathrm{mg}$ dosage as compared to the $100 \%$ that is mandatory in RCTs (AWARD-6). A previous study, on the comparisons between liraglutide and exeOW has yielded a similar conclusion. Indeed, the DURATION-6 RCT showed greater Hablc and weight reduction with liraglutide $1.8 \mathrm{mg}$ compared to ExeOW, ${ }^{27}$ but in RWS the lack of adequate dose escalation of liraglutide resulted in equivalent effectiveness compared to exeOW. ${ }^{38}$ Another highly informative comparison emerged from the comparison of dulaglutide vs exeOW. In this case, there is a complete lack of RCTs with this type of direct head-to-head comparison that however was evaluated in two different network meta-analyses showing equivalent effect on weight and $\mathrm{HbAlc}$ reduction between the two compounds. $^{33,34}$ Conversely, meta-analyses of RWS from different countries showed that dulaglutide was superior to exeOW in the reduction of $\mathrm{HbAlc}$ and body weight. ${ }^{37,39}$ Deeper analyses of this RWS allowed the identification of factors likely associated with these apparent discrepancies, recognizing the culprit in the differences in adherence profiles between the two compounds (measured as persistence in prescription or as refill rate from pharmacy datasets). Indeed, although it is generally recognized that adherence in RWS is much lower than that in the controlled setting of RCTs, this is not the same for all compounds. Adherence has been found to be higher for dulaglutide and lower for exeOW, ${ }^{40-44}$ and this might be related to a simpler and easier device used for injection. ${ }^{45}$ Confirming this hypothesis, sensitivity "as-treated" analyses focused only on those subjects with confirmed prescription of treatments at followup visits (considered as subjects with higher adherence)

Table I Head-to-Head Comparison Between GLP-IRAs Compounds on Different Outcomes, Evaluated in Randomized Controlled Trials (RCTs) and Real-World Studies (RWS)

\begin{tabular}{|c|c|c|c|}
\hline Outcomes & RCTs & RWS & Possible Reason Explaining Differences \\
\hline Hbalc & $\begin{array}{l}\text { Lira } 1.8 \mathrm{mg} \text { and Sema } 1.0 \mathrm{mg}>\mathrm{ExeOW}^{27,28} \\
\text { Dula } 1.5 \mathrm{mg}=\text { Lira } 1.8 \mathrm{mg}^{29} \\
\text { Dula }=\text { ExeOW (from NWMA) } \\
\text { Sema }\left(0.5 \text { or } 1.0 \mathrm{mg} \text { ) }>\text { Dula } 1.5 \mathrm{mg}^{29}\right.\end{array}$ & $\begin{array}{l}\text { Lira }=\text { ExeOW }^{38,82} \\
\text { Dula }>\text { Lira }^{37} \\
\text { Dula }>\text { ExeOW }^{35,37} \\
\text { No RWS }\end{array}$ & \multirow{2}{*}{$\begin{array}{l}\text { - Up-titration in RWS is lower than RCTs }{ }^{37,38} \\
\text { - Overall Adherence in RWS is lower than RCTs }{ }^{37,38,46} \\
\text { - Adherence specifically influenced by compound/devices } \\
\text { (eg, dulaglutide>exeOW) } \\
\text { - Different clinical characteristics between patients enrolled in } \\
\text { RCTs vs those treated with GLP-IRAs in RWS (see Table 2) }\end{array}$} \\
\hline Body Weight & $\begin{array}{l}\text { Lira } 1.8 \mathrm{mg} \text { and Sema } 1.0 \mathrm{mg}>\mathrm{ExeOW}^{27,28} \\
\text { Dula } 1.5 \mathrm{mg}<\text { Lira } 1.8 \mathrm{mg}^{29} \\
\text { Dula }=\text { ExeOW (from NWMA) } \\
\text { Sema }(0.5 \text { or } 1.0 \mathrm{mg})>\text { Dula } 1.5 \mathrm{mg}^{29}\end{array}$ & $\begin{array}{l}\text { Lira }=\text { ExeOW }^{38} \\
\text { Dula }=\text { Lira }^{37} \\
\text { Dula }>\text { ExeOW }^{35,37} \\
\text { No RWS }\end{array}$ & \\
\hline
\end{tabular}

Note: The symbol ">" or "<" are used to describe significant higher or lower efficacy (RCTs) or effectiveness (RWS) between compounds.

Abbreviations: Legend Lira, liraglutide; Sema, semaglutide; ExeOW, exenatide-once-weekly; ExeBID, exenatide-bis-in-die; NWMA, network meta-analyses. 
shows equivalent effectiveness of dulaglutide and exeOW, ${ }^{37}$ as reported in previous network-meta-analyses of RCTs. ${ }^{33,34}$

Differences between effectiveness and efficacy have also been reported in a recent RWS on injectable semaglutide. $^{46}$ The authors found indeed a significant reduction in $\mathrm{HbAlc}$ after treatment with semaglutide $(0.5 \mathrm{mg}$ or $1 \mathrm{mg}$ ) that was lower than that seen in RCTs, ie, SUSTAIN-1. ${ }^{46,47}$ Also in this case, the authors underlined the differences in clinical characteristics of subjects treated in RCTs vs RWS (the latter having a more advanced disease). Moreover, they reported among the likely culprit of the smaller reduction in $\mathrm{HbA} 1 \mathrm{c}$, the role of factors available in RCTs but not in RWS (eg, additional healthcare provider support and scheduled dispensing of therapy) leading to the higher discontinuation rates in RWS as compared to those reported in RCTs. ${ }^{46}$

In Table 2, we have summarized the main differences in clinical characteristics between patients treated with GLP1RAs in RWS and those enrolled in Phase III trials testing the efficacy of GLP-1RAs on glycemic and extra-glycemic effects (excluding CVOTs). Overall, patients in RWS treated with GLP-1RAs compared to those enrolled in phase III RCTs, are more often males, with comparable HbA1c and BMI, but in a later disease stage and often with worst control of other risk factors, such as blood pressure.

\section{GLP-IRAs Combined with Insulin}

Observing the pattern of glucose lowering medications (GLM) prescription over the years, it emerged that GLP-1RAs are often prescribed in combination with basalinsulin. ${ }^{23}$ This combination is a rational and effective treatment option for several patients and can be delivered either by two distinct sub-cutaneous injections (defined as flexible combination) or as a fixed-ratio combination, where the

Table 2 Summary of Main Differences in Clinical Characteristics Between Patients Treated with GLP-IRAs in Real-World Studies (RWS) and Patients Enrolled in Phase III Randomized Controlled Trials (RCTs) Testing Efficacy of GLP-IRAs on Glycemic and Extra-Glycemic Targets

\begin{tabular}{|l|l|}
\hline Characteristics & Patients in RWS vs RCT \\
\hline Age & Older age in RWS \\
Sex & Variable, often more male treated with \\
& GLP-IRA in RWS \\
Disease stage & More advanced disease in RWS \\
HbAlc & No major differences \\
Body Mass Index & No major differences \\
Blood pressure control & Variable, with trend for worse control in RWS \\
\hline
\end{tabular}

same device contains both basal insulin and a GLP-1RA. To date, two different fixed combinations are available (IglarLixi, insulin glargine/lixisenatide and IdegLira, insulin degludec and liraglutide). Although similar, the flexible and fixed approaches have different advantages and limitations. This has been described in a recent RWS, ${ }^{48}$ comparing initiators of GLP-1RAs on top of basal-insulin in either a fixed (IdegLira) or flexible combination. After propensity score matching, yielding two groups of patients with similar characteristics, the study showed that reduction in HbAlc was similar with the fixed vs the flexible combination, while weight loss was greater with the flexible combination. These differences were found to be related to the final dose of basal-insulin and GLP-1RAs. This study therefore showed that, in clinical practice, the advantages of a single-day injection combining GLP-1RAs and basal-insulin, can result in the under-titration of GLP-1RAs for those patients not receiving high basal-insulin (ie, not allowing to increase the GLP-1RAs dosed due to the fixed basal-insulin/GLP-1RAs combination). Yet, to optimize glucose control in patients who do not need substantial weight loss, the fixed combination may represent a simpler approach than the flexible combination.

\section{GLP-IRAs vs Dipeptidyl Peptidase-4 Inhibitor}

RWS can also provide relevant pieces of information for comparisons that have been extensively evaluated in RCTs. For instance, several phase III RCTs have compared the efficacy of GLP-1RAs with that of Dipeptidyl peptidase-4 inhibitor (DPP4i) on HbA1c and weight reduction, consistently showing better performance of GLP-1RAs, as reviewed elsewhere. ${ }^{49}$ In this context, RWS, beyond confirming such results, highlighted how the effectiveness of GLP-1RAs, as compared to that of DPP4i, is more likely to be affected by adherence to treatment. ${ }^{50}$ This is an aspect that requires careful evaluation in clinical practice in order to achieve the same clinical benefit described in RCTs.

\section{GLP-IRAs vs SGLT2i}

The current recommendation to prescribe GLP-1RAs and/ or SGLT-2 $\mathrm{i}$ treatments in the early stages of diabetes brings physicians to the need of choosing frequently which of the two classes of medications to start first. Despite this need, only a few studies have performed a head-to-head comparison between injectable GLP1RAs and SGLT2 $\mathrm{i}^{51}$ with some additional evidence being derived from network meta-analyses. ${ }^{32,52}$ As described in Table 3, the overall results from these RCTs have identified a general greater efficacy on $\mathrm{HbAlc}$ 
Table 3 Head-to-Head Comparison Between GLP-IRAs and SGLT-2i on Different Outcomes, Evaluated in Randomized Controlled Trials (RCTs) and Real-World Studies (RWS)

\begin{tabular}{|c|c|c|c|}
\hline Outcomes & RCTs & RWS & Possible Reason Explaining Differences \\
\hline Hbalc & $\begin{array}{l}\text { Sema } 1.0 \text { mg s.c. > Cana } 300 \mathrm{mg}^{51} \\
\text { (Oral Sema I4mg > Empa } 25 \mathrm{mg}) \\
\text { Lira > SGLT-2i (NWMA) } \\
\text { Long-Acting GLP-IRAs > SGLT-2i } \\
(\text { NWMA) })^{32}\end{array}$ & $\begin{array}{l}\text { No RWS } \\
\text { No RWS } \\
\text { Lira }=\text { Empa }^{54} \\
\text { GLP-IRA (Lira and exeOW) > } \\
\text { Dapa }^{53} \\
\text { GLP-IRA = Cana } 300^{55,56}\end{array}$ & \multirow[t]{2}{*}{$\begin{array}{l}\text { - Up-titration of Lira in RWS is lower than } \\
\text { RCTs } \\
\text { - Higher discontinuation GLP-IRA vs Cana in } \\
\text { RWS }\end{array}$} \\
\hline Body Weight & $\begin{array}{l}\text { Sema } 1.0 \mathrm{mg} \text { s.c. }>\text { Cana } 300^{51} \\
\text { (Oral Sema } 14 \mathrm{mg}=\text { Empa } 25 \mathrm{mg}) \\
\text { Lira } 1.2 \mathrm{mg}<\text { Cana } 300 \mathrm{mg}(\mathrm{NWMA})^{52} \\
\text { Long-Acting GLP-IRAs }>\text { SGLT-2i } \\
\left(\text { NWMA) }{ }^{32}\right.\end{array}$ & $\begin{array}{l}\text { No RWS } \\
\text { No RWS } \\
\text { Lira }=\text { Empa }^{54} \\
\text { GLP-IRA (Lira and exeOW) = } \\
\text { Dapa }{ }^{53} \\
\text { GLP-IRA = Cana } 300 \mathrm{mg}^{55,56}\end{array}$ & \\
\hline
\end{tabular}

Note: The symbol ">” or "<" are used to describe significant higher or lower efficacy or effectiveness between compounds.

Abbreviations: Lira, liraglutide; Sema, semaglutide; ExeOW, exenatide-once-weekly; ExeBID, exenatide-bis-in-die; Cana, canagliflozin; Dapa, dapagliflozin; NWMA, network meta-analyses.

reduction and on weight reduction of injectable longacting GLP-1RAs. The comparison of GLP-1RAs vs SGLT-2 $\mathrm{i}$ in RWS has been conducted mainly combining different GLP-1RAs. In this context, RWS have confirmed the greater reduction in $\mathrm{HbAlc}$ when comparing GLP1RAs (mainly liraglutide and exenatide-OW) with dapagliflozin ${ }^{53}$ but not compared with emapagliflozin ${ }^{54}$ or canagliflozin. ${ }^{55,56}$ The same comparisons have not identified major differences in weight changes between the two classes of drugs. One possible reason that might explain these results might be the lower adherence for GLP-1RAs found in RWS. ${ }^{53-56}$ For instance, the adherence to treatment was higher for canagliflozin compared to GLP-1RAs (liraglutide and exenatide) in different studies. ${ }^{55,56}$ However, it should be noted that these findings should not be extrapolated to GLP-1RAs that were approved by drug regulatory agencies only recently (eg, dulaglutide and semaglutide) and for which similar RWS are not yet published.

\section{Perspective}

RWS showed different performance of some GLP-1RAs as compared to that expected from RCTs (efficacy vs effectiveness). Since these differences seems to be attributable to modifiable factors (eg, adequate up-scaling and adherence) it will be essential to consider and address these issues when treating subjects with GLP-1RAs. At the same time, these aspects highlight the importance of measuring and reporting performances of interventions (including but not limited to GLP-1RAs) in clinical practice.

\section{Effectiveness in Special Populations}

Another opportunity provided by RWS is to evaluate the effectiveness of GLP-1RAs in those populations that are generally under-represented in RCTs, such as the elderly, subjects with CKD and other comorbidities, or non-obese individuals. ${ }^{23}$ To this end, our group has recently shown how treatment with dulaglutide, over a median observation time of 13 months, exerted an equivalent reduction in $\mathrm{HbAlc}$ and body weight in obese and non-obese subjects, the latter being more than a quarter of the entire population treated with the GLP-1RAs in real-world settings. These results therefore confirmed, in a longer follow-up and in a real-world setting, the results reported in the AWARD trial program. ${ }^{57}$ Importantly, our study found no evidence of interaction with age, or presence of cardiovascular or renal disease, meaning that the effectiveness of dulaglutide on $\mathrm{HbAlc}$ is equivalent regardless of presence or absence of age $>75$ years old, or cardiovascular disease or diabetic kidney disease. At the same, while confirming results of post-hoc RCT analyses, ${ }^{58,59}$ these studies also highlight the important role of persistence and adherence to treatment as an important determinant of its effectiveness. ${ }^{60}$ Indeed, although we found overall high persistence (around 85\% after around 13 months), those who discontinued the treatment had a striking worsening glucose control and regain of weight. ${ }^{23}$ 
Another limitation in RCTs is the unbalance in representation of race/ethnic subgroups. Indeed some groups, eg, Hispanic, Asian, Black or African-American subjects, despite having higher prevalence of diabetes compared to White subjects, ${ }^{61}$ are generally less represented in RCTs and in large CVOTs. While stratification of participants according to race or ethnic groups have not identified significant differences in the cardiovascular efficacy of GLP-1RAs, ${ }^{6-9}$ given the under-representation of these populations in CVOTs, it is currently unclear whether GLP-1RAs reduce cardiovascular risk in specific race/ethnic groups (eg, black patients). ${ }^{62}$ Moreover, it is unknown whether this under-representation in RCTs affects routine clinical practice and GLP-1RAs prescription. In this context, RWS have shown how subjects belonging to minorities have lower probability to achieve appropriate glucose control and control of other cardiovascular risk factors. ${ }^{63-66}$ Moreover, race has been found to be one of the factors influencing the selection of first injectable treatment (GLP-1RAs vs insulin) in US patients with type 2 diabetes. ${ }^{67}$ This is also supported by a recent secondary analyses of the Look-AHEAD (Action for Health in Diabetes) trial, showing racial and social-economic status disparities in the use of innovative glucose lowering medications (ie, DPP-4i, or SGLT-2i or GLP-1RAs). ${ }^{68}$ Beyond the analyses of pattern of GLP-1RAs use by race/ethnic group, there is a lack of data on RWS evaluating the effectiveness of GLP-1RAs in these populations, underlining therefore the need for further RCTs and RWS on this aspect.

\section{Perspective}

RWS confirm the transferability of results seen in RCTs to populations less represented in these trials, such as the elderly or non-obese individuals or those with diabetic kidney disease. Analyses of data from clinical practice also show the presence of racial and social-economic status disparities in the initiation of innovative treatments, including GLP-1RAs. RWS therefore highlight the urgent need to address those aspects that might be responsible for disparities and to conduct more studies to evaluate the effectiveness in these populations under-represented in clinical trials.

\section{Effectiveness on Hard Cardiovascular and Renal Outcomes}

The great interest towards GLP-1RAs has been advocated by the beneficial effects of this class of medications on major cardiovascular outcomes, mortality and, to a lesser extent, renal outcomes. ${ }^{10}$ Given the aforementioned differences between CVOTs and RWS, it is therefore essential to evaluate whether similar results are confirmed in the observational clinical practice setting. At the same time, in this case the challenges to conduct a rigorous and reliable observational study are manifold. One major difference in the parallelism between CVOTs and RWS on hard outcomes is that, since CVOTs have been initially designed to test the cardiovascular safety of new glucose-lowering medications, these are generally tested against placebo. On the contrary, outside RCTs, subjects are never treated with placebo, and therefore RWS most of the time compared GLP-1RAs (or other drugs of interest) with an active comparator, thus yielding more clinically-relevant information. Indeed, when a physician needs to add a GLM to improve patient conditions, he or she needs to choose between two different treatments, while the choice is rarely for a treatments vs no-treatment (that would be equivalent of a placebo-controlled comparison).

To this end, some RWS have tested the effectiveness of GLP-1RAs on hard outcomes. One of the first studies analysed data collected from over 3.5 million patients registered from over 640 UK general practitioners. ${ }^{69}$ After matching for age, BMI, sex, duration of diabetes and smoking status, the authors compared 8345 GLP-1RAs users with 16,541 controls (ie, subjects unexposed to GLP-1RAs matched with similar index date to avoid immortal time bias). The primary outcome of this study was overall mortality that was found to be significantly lower among GLP-1RA users (adjusted incidence rate ratio: $0.64,95 \% \mathrm{CI}: 0.56-0.74$ ) and not influenced by baseline cardiovascular risk, age or $\mathrm{HbAlc}$ level at baseline. ${ }^{70}$ Two studies have recently interrogated healthcare clinical databases from Denmark and Sweden ${ }^{71}$ and form North-East Italy ${ }^{72}$ to investigate whether use of GLP-1RAs was associated with better cardiovascular outcomes compared to DPP4i. The first study was focused on liraglutide only and involved 23,402 subjects in each group, while the second included 2807 subjects per group treated with a miscellaneous of different GLP-1RAs (liraglutide, dulaglutide, exenatide and lixisenatide) and DPP-4i. Both studies used a new-user design and applied propensity score matched analyses to address channelling bias. Both studies confirmed that users of GLP-1RAs had lower incidence of MACE and lower all-cause and cardiovascular mortality than subjects with similar clinical characteristics treated with DPP4i. ${ }^{71,72}$ Another RWS from Taiwan's nation-wide population-base cohort found that, after propensity score 
matching, prevalent users of GLP-1RAs had lower MACE events compared to prevalent users of DPP4i, sulfonylurea or insulin. ${ }^{73}$

The benefit of GLP-1RAs on diabetic kidney disease described in RCTs has been found to be mainly related to reduction in albumin excretion. ${ }^{10}$ Beyond this benefit, a recent RWS has also investigated the effect of GLP1RAs on a composite outcome of serious renal events (including renal replacement therapy, death from renal causes and hospitalization for renal events). ${ }^{74}$ This study was conducted on 38,731 new-users, matched by propensity score with the same number of controls (new users of DPP4i). It found lower incidence of the composite outcome (H.R. 0.76, 95\% C.I. 0.68-0.85), as well as of renal replacement and hospitalization for renal events, in the subjects treated with GLP-1RAs. Thanks to the inclusion of a large population and to the link with Scandinavian health registry (proving accurate classification of the outcomes), this RWS allowed to overcome the limits or RCTs. In fact, these outcomes were either not reported or have a limited number of events in most RCTs, $9,75,76$ thereby yielding uncertain results.

Finally, our group has recently explored the comparison between GLP-1RAs and SGLT-2i on the incidence of cardiovascular events in a real-world setting. ${ }^{77}$ Of note, given their beneficial effects, these two classes of drugs are currently recommended as second-line treatment for the management of T2D and should be used with a treat-to-benefit approach for all patients on secondary cardiovascular prevention or with very-high cardiovascular risk. ${ }^{19}$ Despite this recommendation, to date, no RCT has directly compared the cardiovascular efficacy of these two classes of drugs. Therefore, RWS provide a great opportunity to start exploring this comparison. By means of the analyses of electronic health data linked with administrative database and hospital discharge codes, we analysed 4298 new-users of SGLT-2i matched with 4298 new-users of GLP-1RAs. The SGLT-2i users experienced a lower rate of 3-point MACE (Hazard Ratio, H.R. 0.68, 95\% C.I. 0.61-0.99, p=0.043) as well as lower rate of myocardial infarction (H.R.: 0.72; 95\% CI 0.53-0.98; $\mathrm{p}=0.035$ ) and hospitalization for heart failure (H.R. 0.59; 95\% CI 0.35-0.99; $\mathrm{p}=0.048$ ). These results were confirmed also in the as-treated analyses (ie, including only those subjects that were persistently on treatment with each drug). Of course, the level of evidence that can be derived from this study is moderate, because of residual confounding. Although such results absolutely require confirmation before being incorporated in clinical decision- making, they highlight the role of RWS beyond that of validation of results of RCTs. Given that EASD/ADA guidelines generally suggest GLP-1RAs are more active against atherosclerotic disease than SGLT-2i, the finding of a lower 3-P MACE among new users of SGLT2 $\mathrm{i}$ versus new users of GLP-1RAs was unexpected and identifies an area where RWS and RCTs are not consistent. There may be several reasons to explain such inconsistency. In addition to the residual confounding driven by the non-randomized nature of the study (see below), time overall follow-up (18 months in the ITT and 13 months in the AT) was probably short to allow for the anti-atherosclerotic effects of GLP-1RAs to emerge.

Beyond the head-to-head comparison between GLP1RA and SGLT-2i, there is an increasing interest in the evaluation on whether the combination of these two classes of drugs will provide additional benefit on cardiovascular outcomes beyond the use of only one of the two classes. Although there is currently no result from RCTs testing this highly relevant question, the increasing combined use of the two in routine clinical practice will allow exploring of this hypothesis in a future RWS.

\section{Perspective}

RWS overall support the cardiovascular benefits of GLP1RAs shown in RCTs. Moreover, the superiority, in terms of reduction of cardiovascular events with the use of GLP1RAs as compared to DPP4i found in different studies, not only reinforce the current recommendations of guidelines, but also advocate for the urgent need to change prescription patterns of GLMs, where DPP4i are still largely more used than GLP-1RAs. Moreover, RWS suggest that GLP1RAs might provide beneficial effects on severe renal outcomes that have not been identified in RCTs. They also suggest that a direct comparison between GLP-1RAs and of SGLT-2 $\mathrm{i}$ on cardiovascular outcomes might favour of SGLT-2i, but here further studies are warranted to explore this relevant clinical question.

\section{Quality Assessment and Limitations of Real-World Studies Assessing Treatment Effectiveness}

In order to interpret correctly RWS, it is essential to be aware of several limitations that can influence the results of such studies. ${ }^{11,12,78,79}$ Indeed, even in the most rigorous RWS conducted with up-to-date statistical approaches (like those described in this review), residual bias (ie, systematic error) cannot be completely ruled out. With 
Table 4 Main Biases Domains to Be Considered When Assessing Quality of Non-Randomized Studies

\begin{tabular}{|c|c|c|}
\hline $\begin{array}{l}\text { Biases } \\
\text { Domains }\end{array}$ & Description & Details/Example \\
\hline $\begin{array}{l}\text { Confounding } \\
\text { Biases }\end{array}$ & $\begin{array}{l}\text { Presence of factors that may influence the results such that } \\
\text { the observed association between intervention and outcomes } \\
\text { differs from the causal effect. }\end{array}$ & $\begin{array}{l}\text { May be referred to residual confounding (not appropriate } \\
\text { analyses of known and measured confounders) or to } \\
\text { unmeasured confounding (factors not measured at all or not } \\
\text { included in the analyses). }\end{array}$ \\
\hline Selection Biases & $\begin{array}{l}\text { Exclusion of subjects (or events/outcomes or follow-up time) } \\
\text { that lead to systematic errors in the estimated association } \\
\text { between intervention and outcomes. }\end{array}$ & $\begin{array}{l}\text { Bias in selection of subjects can be related to both } \\
\text { intervention and outcome. Some example are immortal-time- } \\
\text { bias, or bias arising from exclusion of subjects with missing } \\
\text { data. }\end{array}$ \\
\hline $\begin{array}{l}\text { Information } \\
\text { Biases } \\
\text { (measurement } \\
\text { bias) }\end{array}$ & $\begin{array}{l}\text { Presence of Misclassification of intervention status or } \\
\text { outcomes. }\end{array}$ & $\begin{array}{l}\text { Misclassification of intervention status might happen on } \\
\text { retrospective cohort studies if availability of information on } \\
\text { interventions are influenced by outcomes (a.k.a. recall bias). } \\
\text { Misclassification of outcomes (detection bias), eg, when } \\
\text { intensities of observations/measurement of outcomes differs } \\
\text { between the intervention groups. }\end{array}$ \\
\hline Reporting Biases & $\begin{array}{l}\text { Selection of the reported results arising from a desire for } \\
\text { findings to merit publication. }\end{array}$ & $\begin{array}{l}\text { Biases arise when the selection of results is based on P-values, } \\
\text { magnitude or direction of the estimated effect of intervention. } \\
\text { Might concern selection of outcomes, selection of the } \\
\text { analyses, or selective reporting of a subgroup of participants. }\end{array}$ \\
\hline
\end{tabular}

little doubt, one of the most important bias in retrospective observational studies testing effectiveness of interventions is the lack of randomization. This can lead to confounding by indication, or channelling bias, meaning that the exposure to the drugs being tested are influenced by patients' characteristics or comorbidities and such characteristics then drive differential outcome more than the drugs themselves. Several reliable statistical methods have been developed to specifically address this bias, such as multivariate adjusted analyses and propensity score based analyses. Yet, these approaches can balance only measured and measurable confounding factors, such that RWS cannot exclude the possibility that one or more un-measured, un-measurable or unknown independent factor could bias the results. Another critical aspect in RWS is the burden of missing data. Several techniques, such as multiple imputation, can avoid excluding subjects with missing data thus reducing generalizability of results. ${ }^{80}$ Yet, imputation of missing data can be seen as an artificial inflation of available data leading to overfitting of the results.

Another issue to be considered are immortal-time bias (biased exposure status based on future information, ie, a period of time during which the person is event-free by study design), time-lag bias (interval time immediately after drug initiation in which the outcomes cannot be attributable to the drugs), or possible over-adjustment (ie, when analyses are adjusted or matched for factors that are mediators of the effect of interest and not confounding factors). ${ }^{78}$

Table 4 summarizes the main limitations and biases that should be evaluated when assessing the quality of RWS, and that are described more in details in other excellent reviews, ${ }^{11,12,78,79}$ and in Cochrane textbooks. ${ }^{81}$ Researcher and clinicians evaluating results from RWS should consider the importance of quality assessment of risk of bias. This can be done using specific tools, such as the Risk Of Bias In Non-randomized Studies of Interventions (ROBINS-I tool), as recommended by the Cochrane group. ${ }^{81}$ This tool evaluates seven domains: bias due to confounding, bias to selection of participants, bias in classification of interventions, bias due to deviations from intended interventions, bias due to missing data, bias in measurement outcomes and bias in selection of the reported results. The RWS testing effectiveness of GLP-1RAs included in this review have been found to have mainly low-to-moderate risk of bias in these domains. One of the two most common limitations found in the study was the possible deviation from intended intervention (eg, information on adherence noncomparable to that of well conducted RCTs) and the lack of a pre-specified protocol with details on planned analyses that might lead to selection of the reported results. 
As previously discussed in Glycemic and ExtraGlycemic Effectiveness, the insurance coverage of these costly new agents (GLP1RA and SGLT2i) can influence the prescription and adherence to these drugs in RWS. Therefore, is it highly important to take this important aspect into consideration when analysing results from RWS. If not appropriately accounted for, lack of adherence and discontinuation may bias results away from those of RCTs. This aspect deserves specific attention in RWS conducted in countries without universal health coverage (eg, the US), while in countries where all citizens have the same health coverage regardless of socio-economic-status this aspect is less likely to bias RWS (eg, in Italy).

Altogether, the evaluation and consideration of all these important limitations and potential biases with the implementation of rigorous and appropriate research methods is pivotal to allow RWS to play the role they deserve in diabetes research, complementary to that played by RCTs. $^{79}$ The role of RWS has also been recognized by the Food and Drug Administration (FDA) and European Medicines Agency (EMA), that have provided guidelines for the use of RWS to support regulatory decision-making for medical treatments and devices (www.fda.gov and http://www.ema.europa.eu/).

\section{Conclusions}

In conclusion, data from clinical practice supports the role of GLP-1RAs in the current armamentarium for the treatment of T2D as recommended in the EASD/ADA treatment algorithm. ${ }^{19}$ They also highlight the unmet need that RCTs should enrol subjects with clinical characteristics more similar to those of patients routinely attending diabetes clinics. Physicians should consider modifiable factors (eg, up-titration or adherence) that might influence effectiveness of GLP-1RA. Finally, RWS consistently reported better cardiovascular and renal outcomes of users of GLP-1RAs as compared to DPP-4 inhibitors users, and highlights the needs for further RWS comparing GLP-1RAs and SGLT-2i (and their combinations) on cardiovascular outcomes, that should eventually be confirmed by dedicated RCTs.

\section{Authorship}

All authors met the International Committee of Medical Journal Editors (ICMJE) for authorship and approved the final version of the manuscript.

\section{Abbreviations}

ADA, American Diabetes Association; CVOT, cardiovascular outcome trial; DPP4i, dipeptidyl peptidase-4 inhibitor; EASD, European Association for the Study of Diabetes; ExeOW, exenatide once-weekly; GLM, glucose lowering medications; GLP-1RAs, glucagon-likepeptide-1 receptor agonists; H.R., hazard ratio; MACE, major adverse cardiovascular events; RCTs, randomized controlled trials; RWS, real-world studies; SGLT-2i, sodium/glucose cotransporter 2-inhibitors; T2D, type 2 Diabetes.

\section{Disclosure}

MLM received grant support, lecture or consultant fees from: Servier, Amryt and SLAPharma, not related to the submitted work. . AA received research grants, lecture or advisory board fees from: Merck Sharp \& Dome, AstraZeneca, Novartis, Boehringer-Ingelheim, Sanofi, Mediolanum, Janssen, Novo Nordisk, Lilly, Servier, and Takeda, not related to the submitted work. GPF received lecture fees or grant support from: Abbott, AstraZeneca, Boehringer, Lilly, Merck-Sharp-Dome, Mundipharma, Novartis, Novo Nordisk, Sanofi, Servier, not related to the submitted work. The authors report no other potential conflicts of interest for this work.

\section{References}

1. Andersen A, Lund A, Knop FK, Vilsboll T. Glucagon-like peptide 1 in health and disease. Nat Rev Endocrinol. 2018;14(7):390-403. doi:10.1038/s41574-018-0016-2

2. Skov J. Effects of GLP-1 in the kidney. Rev Endocr Metab Disord. 2014;15(3):197-207. doi:10.1007/s11154-014-9287-7

3. Pantalone KM, Munir K, Hasenour CM, et al. Cardiovascular outcomes trials with glucagon-like peptide-1 receptor agonists: a comparison of study designs, populations and results. Diabetes Obes Metab. 2020. doi:10.1111/dom.14165

4. Bonaventura A, Carbone S, Dixon DL, Abbate A, Montecucco F. Pharmacologic strategies to reduce cardiovascular disease in type 2 diabetes mellitus: focus on SGLT-2 inhibitors and GLP-1 receptor agonists. J Intern Med. 2019;286(1):16-31. doi:10.1111/joim.12890

5. Heuvelman VD, Van Raalte DH, Smits MM. Cardiovascular effects of glucagon-like peptide 1 receptor agonists: from mechanistic studies in humans to clinical outcomes. Cardiovasc Res. 2020;116(5):916-930.

6. Marso SP, Daniels GH, Brown-Frandsen K, et al. Liraglutide and cardiovascular outcomes in type 2 diabetes. $N$ Engl J Med. 2016;375 (4):311-322. doi:10.1056/NEJMoa1603827

7. Gerstein HC, Colhoun HM, Dagenais GR, et al. Dulaglutide and cardiovascular outcomes in type 2 diabetes (REWIND): a double-blind, randomised placebo-controlled trial. Lancet. 2019;394(10193):121-130.

8. Hernandez AF, Green JB, Janmohamed S, et al. Albiglutide and cardiovascular outcomes in patients with type 2 diabetes and cardiovascular disease (harmony outcomes): a double-blind, randomised placebo-controlled trial. Lancet. 2018;392(10157):1519-1529. 
9. Marso SP, Bain SC, Consoli A, et al. Semaglutide and cardiovascular outcomes in patients with type 2 diabetes. $N$ Engl J Med. 2016;375 (19):1834-1844. doi:10.1056/NEJMoa1607141

10. Kristensen SL, Rorth R, Jhund PS, et al. Cardiovascular, mortality, and kidney outcomes with GLP-1 receptor agonists in patients with type 2 diabetes: a systematic review and meta-analysis of cardiovascular outcome trials. Lancet Diabetes Endocrinol. 2019;7(10): 776-785. doi:10.1016/S2213-8587(19)30249-9

11. Gerstein HC, McMurray J, Holman RR. Real-world studies no substitute for RCTs in establishing efficacy. Lancet. 2019;393(1016 8):210-211.

12. Yang W, Zilov A, Soewondo P, Bech OM, Sekkal F, Home PD. Observational studies: going beyond the boundaries of randomized controlled trials. Diabetes Res Clin Pract. 2010;88(Suppl 1):S3-9. doi:10.1016/S0168-8227(10)70002-4

13. Stuart EA, Bradshaw CP, Leaf PJ. Assessing the generalizability of randomized trial results to target populations. Prev Sci. 2015;16 (3):475-485. doi:10.1007/s11121-014-0513-z

14. Malmivaara A. Generalizability of findings from randomized controlled trials is limited in the leading general medical journals. J Clin Epidemiol. 2019;107:36-41. doi:10.1016/j.jclinepi.2018.11.014

15. Unger JM, Cook E, Tai E, Bleyer A. The role of clinical trial participation in cancer research: barriers, evidence, and strategies. Am Soc Clin Oncol Educ Book. 2016;35:185-198. doi:10.1200/ EDBK 156686

16. Sciannameo V, Berchialla P, Orsi E, et al. Enrolment criteria for diabetes cardiovascular outcome trials do not inform on generalizability to clinical practice: the case of glucagon-like peptide-1 receptor agonists. Diabetes Obes Metab. 2020;22(5):817-827. doi:10.11 11/dom. 13962

17. Boye KS, Riddle MC, Gerstein HC, et al. Generalizability of glucagon-like peptide-1 receptor agonist cardiovascular outcome trials to the overall type 2 diabetes population in the United States. Diabetes Obes Metab. 2019;21(6):1299-1304. doi:10.1111/dom.13649

18. Collins R, Bowman L, Landray M, Peto R. The magic of randomization versus the myth of real-world evidence. $N$ Engl J Med. 2020;382 (7):674-678. doi:10.1056/NEJMsb1901642

19. Buse JB, Wexler DJ, Tsapas A, et al. 2019 update to: management of hyperglycemia in type 2 diabetes, 2018. A consensus report by the American diabetes association (ADA) and the European association for the study of diabetes (EASD). Diabetes Care. 2020;43(2): 487-493.

20. Montvida O, Shaw J, Atherton JJ, Stringer F, Paul SK. Long-term trends in antidiabetes drug usage in the U.S.: real-world evidence in patients newly diagnosed with type 2 diabetes. Diabetes Care. 2018;41(1):69-78. doi:10.2337/dc17-1414

21. Fadini GP, Frison V, Simioni N, et al. Changes in the prescription of glucose-lowering medications in patients with type 2 diabetes mellitus after a cardiovascular event: a call to action from the DATAFILE study. J Am Heart Assoc. 2019;8(14):e012244. doi:10.1161/JAHA. 119.012244

22. Franch-Nadal J, Mata-Cases M, Ortega E, et al. Glucagon-like peptide-1 receptor agonists in patients with type 2 diabetes: prescription according to reimbursement constraints and guideline recommendations in catalonia. J Clin Med. 2019;8(9):1389.

23. Fadini GP, Frison V, Rigato M, et al. Trend 2010-2018 in the clinical use of GLP-1 receptor agonists for the treatment of type 2 diabetes in routine clinical practice: an observational study from Northeast Italy. Acta Diabetol. 2020;57(3):367-375. doi:10.1007/s00592-019-01445-z

24. Knudsen JS, Baggesen LM, Lajer M, et al. Changes in SGLT2i and GLP-1RA real-world initiator profiles following cardiovascular outcome trials: a Danish nationwide population-based study. PLoS One. 2020;15(3):e0229621. doi:10.1371/journal.pone.0229621

25. Revicki DA, Frank L. Pharmacoeconomic evaluation in the real world. Effectiveness versus efficacy studies. PharmacoEconomics. 1999;15(5):423-434. doi:10.2165/00019053-199915050-00001
26. Madsbad S. Review of head-to-head comparisons of glucagon-like peptide-1 receptor agonists. Diabetes Obes Metab. 2016;18 (4):317-332. doi:10.1111/dom.12596

27. Buse JB, Nauck M, Forst T, et al. Exenatide once weekly versus liraglutide once daily in patients with type 2 diabetes (DURATION-6): a randomised, open-label study. Lancet. 2013;381 (9861):117-124. doi:10.1016/S0140-6736(12)61267-7

28. Ahmann AJ, Capehorn M, Charpentier G, et al. Efficacy and safety of once-weekly semaglutide versus exenatide ER in subjects with type 2 diabetes (SUSTAIN 3): a 56-week, open-label, randomized clinical trial. Diabetes Care. 2018;41(2):258-266. doi:10.2337/dc17-0417

29. Dungan KM, Povedano ST, Forst T, et al. Once-weekly dulaglutide versus once-daily liraglutide in metformin-treated patients with type 2 diabetes (AWARD-6): a randomised, open-label, phase 3, non-inferiority trial. Lancet. 2014;384(9951):1349-1357. doi:10.101 6/S0140-6736(14)60976-4

30. Wysham C, Blevins T, Arakaki R, et al. Efficacy and safety of dulaglutide added onto pioglitazone and metformin versus exenatide in type 2 diabetes in a randomized controlled trial (AWARD-1). Diabetes Care. 2014;37(8):2159-2167. doi:10.2337/dc13-2760

31. Pratley RE, Aroda VR, Lingvay I, et al. Semaglutide versus dulaglutide once weekly in patients with type 2 diabetes (SUSTAIN 7): a randomised, open-label, phase $3 \mathrm{~b}$ trial. Lancet Diabetes Endocrinol. 2018;6(4):275-286. doi:10.1016/S2213-8587(18)30024-X

32. Hussein H, Zaccardi F, Khunti K, et al. Efficacy and tolerability of sodium-glucose co-transporter-2 inhibitors and glucagon-like peptide-1 receptor agonists: a systematic review and network meta-analysis. Diabetes Obes Metab. 2020;22(7):1035-1046. doi:10.1111/dom.14008

33. Htike ZZ, Zaccardi F, Papamargaritis D, Webb DR, Khunti K, Davies MJ. Efficacy and safety of glucagon-like peptide-1 receptor agonists in type 2 diabetes: a systematic review and mixed-treatment comparison analysis. Diabetes Obes Metab. 2017;19(4):524-536. doi:10.1111/dom.12849

34. Orme ME, Nguyen H, Lu JY, Thomas SA. Comparative effectiveness of glycemic control in patients with type 2 diabetes treated with GLP-1 receptor agonists: a network meta-analysis of placebo-controlled and active-comparator trials. Diabetes Metab Syndr Obes. 2017;10:111-122. doi:10.2147/DMSO.S116810

35. Taylor SI. GLP-1 receptor agonists: differentiation within the class. Lancet Diabetes Endocrinol. 2018;6(2):83-85. doi:10.1016/S22138587(17)30413-8

36. Zweck E, Roden M. GLP-1 receptor agonists and cardiovascular disease: drug-specific or class effects? Lancet Diabetes Endocrinol. 2019;7(2):89-90. doi:10.1016/S2213-8587(18)30351-6

37. Morieri ML, Rigato M, Frison V, et al. Effectiveness of dulaglutide vs liraglutide and exenatide once-weekly. A real-world study and meta-analysis of observational studies. Metabolism. 2020;106:154190. doi:10.1016/j.metabol.2020.154190

38. Fadini GP, Bonora BM, Lapolla A, et al. Comparative effectiveness of exenatide once-weekly versus liraglutide in routine clinical practice: a retrospective multicentre study and meta-analysis of observational studies. Diabetes Obes Metab. 2019;21(5):1255-1260. doi:10.1111/ dom. 13623

39. Unni S, Wittbrodt E, Ma J, et al. Comparative effectiveness of once-weekly glucagon-like peptide-1 receptor agonists with regard to 6-month glycaemic control and weight outcomes in patients with type 2 diabetes. Diabetes Obes Metab. 2018;20(2):468-473. doi:10.1111/dom.13107

40. Federici MO, McQuillan J, Biricolti G, et al. Utilization patterns of glucagon-like peptide-1 receptor agonists in patients with type 2 diabetes mellitus in Italy: a retrospective cohort study. Diabetes Ther. 2018;9(2):789-801.

41. Otto T, Myland M, Jung H, Lebrec J, Richter H, Norrbacka K. Utilization patterns of glucagon-like peptide-1 receptor agonists in patients with type 2 diabetes mellitus in Germany: a retrospective cohort study. Curr Med Res Opin. 2019;35(5):893-901. doi:10.1080/ 03007995.2018.1538011 
42. Divino V, Boye KS, Lebrec J, DeKoven M, Norrbacka K. GLP-1 RA treatment and dosing patterns among type 2 diabetes patients in six countries: a retrospective analysis of pharmacy claims data. Diabetes Ther. 2019;10(3):1067-1088.

43. Alatorre C, Fernandez Lando L, Yu M, et al. Treatment patterns in patients with type 2 diabetes mellitus treated with glucagon-like peptide-1 receptor agonists: higher adherence and persistence with dulaglutide compared with once-weekly exenatide and liraglutide. Diabetes Obes Metab. 2017;19(7):953-961. doi:10.1111/dom.12902

44. Brown RE, Abitbol A, Bajaj HS, et al. Patient reported outcomes following initiation of glucagon-like peptide-1 receptor agonists in patients with type 2 diabetes in a specialist endocrinology practice of the LMC diabetes registry: the PROGRESS-diabetes study. Diabetes Res Clin Pract. 2019;156:107820. doi:10.1016/j.diabres.2019.107820

45. Amblee A. Mode of administration of dulaglutide: implications for treatment adherence. Patient Prefer Adherence. 2016;10:975-982. doi:10.2147/PPA.S82866

46. Brown RE, Bech PG, Aronson R. Semaglutide once weekly in people with type 2 diabetes: real-world analysis of the Canadian LMC diabetes registry (SPARE study). Diabetes Obes Metab. 2020. doi:10.1111/dom.14117

47. Sorli C, Harashima SI, Tsoukas GM, et al. Efficacy and safety of once-weekly semaglutide monotherapy versus placebo in patients with type 2 diabetes (SUSTAIN 1): a double-blind, randomised, placebo-controlled, parallel-group, multinational, multicentre phase 3a trial. Lancet Diabetes Endocrinol. 2017;5(4):251-260. doi:10.10 16/S2213-8587(17)30013-X

48. Morieri ML, Rigato M, Frison V, et al. Fixed versus flexible combination of GLP-1 receptor agonists with basal insulin in type 2 diabetes: a retrospective multicentre comparative effectiveness study. Diabetes Obes Metab. 2019;21(11):2542-2552.

49. Gilbert MP, Pratley RE. GLP-1 analogs and DPP-4 inhibitors in type 2 diabetes therapy: review of head-to-head clinical trials. Front Endocrinol (Lausanne). 2020;11:178. doi:10.3389/fendo.2020.00178

50. Carls GS, Tan R, Zhu JY, et al. Real-world weight change among patients treated with glucagon-like peptide-1 receptor agonist, dipeptidyl peptidase- 4 inhibitor and sulfonylureas for type 2 diabetes and the influence of medication adherence. Obes Sci Pract. 2017;3 (3):342-351. doi:10.1002/osp4.116

51. Lingvay I, Catarig AM, Frias JP, et al. Efficacy and safety of once-weekly semaglutide versus daily canagliflozin as add-on to metformin in patients with type 2 diabetes (SUSTAIN 8): a double-blind, phase 3b, randomised controlled trial. Lancet Diabetes Endocrinol. 2019;7(11):834-844. doi:10.1016/S2213-8587 (19)30311-0

52. Lorenzi M, Ploug UJ, Langer J, Skovgaard R, Zoratti M, Jansen J. Liraglutide versus SGLT-2 inhibitors in people with type 2 diabetes: a network meta-analysis. Diabetes Ther. 2017;8(1):85-99. doi:10.10 07/s13300-016-0217-4

53. Fadini GP, Sciannameo V, Franzetti I, et al. Similar effectiveness of dapagliflozin and GLP-1 receptor agonists concerning combined endpoints in routine clinical practice: a multicentre retrospective study. Diabetes Obes Metab. 2019;21(8):1886-1894.

54. Grabarczyk TR, Wissman NK. Weight outcomes with empagliflozin as compared with liraglutide in veterans with type 2 diabetes mellitus. Ann Pharmacother. 2020;54(10):981-987. doi:10.1177/106 0028020915791

55. Coleman CI, Pandya S, Wang L, et al. Treatment patterns, glycemic control and bodyweight with canagliflozin $300 \mathrm{mg}$ versus GLP1RAs in type II diabetes patients. J Comp Eff Res. 2019;8(11):889-905. doi:10.2217/cer-2019-0002

56. Wysham $\mathrm{CH}$, Pilon D, Ingham M, et al. Quality goal attainment and maintenance in patients with type II diabetes mellitus initiated on canagliflozin or a glucagon-like peptide-1 receptor agonist in an actual practice setting. Curr Med Res Opin. 2018;34(6):1125-1133. doi:10.1080/03007995.2018.1454417
57. Gentilella R, Sesti G, Vazquez L, et al. Dulaglutide is an effective treatment for lowering HbAlc in patients with type 2 diabetes regardless of body mass index. Diabetes Obes Metab. 2019;21 (12):2660-2666. doi:10.1111/dom.13853

58. Boustani MA, Pittman I, Yu M, Thieu VT, Varnado OJ, Juneja R. Similar efficacy and safety of once-weekly dulaglutide in patients with type 2 diabetes aged $>/=65$ and $<65$ years. Diabetes Obes Metab. 2016;18(8):820-828.

59. Gallwitz B, Dagogo-Jack S, Thieu V, et al. Effect of once-weekly dulaglutide on glycated haemoglobin (HbAlc) and fasting blood glucose in patient subpopulations by gender, duration of diabetes and baseline HbA1c. Diabetes Obes Metab. 2018;20(2):409-418. doi:10.1111/dom.13086

60. Kirkman MS, Rowan-Martin MT, Levin R, et al. Determinants of adherence to diabetes medications: findings from a large pharmacy claims database. Diabetes Care. 2015;38(4):604-609.

61. Ferdinand KC, Nasser SA. Racial/ethnic disparities in prevalence and care of patients with type 2 diabetes mellitus. Curr Med Res Opin. 2015;31(5):913-923. doi:10.1185/03007995.2015.1029894

62. Mishriky BM, Powell JR, Wittwer JA, et al. Do GLP-1RAs and SGLT-2is reduce cardiovascular events in black patients with type 2 diabetes? A systematic review and meta-analysis. Diabetes Obes Metab. 2019;21(10):2274-2283. doi:10.1111/dom.13805

63. Pantalone KM, Misra-Hebert AD, Hobbs TM, et al. The probability of A1C goal attainment in patients with uncontrolled type 2 diabetes in a large integrated delivery system: a prediction model. Diabetes Care. 2020;43(8):1910-1919. doi:10.2337/dc19-0968

64. Tran AT, Berg TJ, Gjelsvik B, et al. Ethnic and gender differences in the management of type 2 diabetes: a cross-sectional study from Norwegian general practice. BMC Health Serv Res. 2019;19(1):904. doi:10.1186/s12913-019-4557-4

65. Campbell JA, Walker RJ, Smalls BL, Egede LE. Glucose control in diabetes: the impact of racial differences on monitoring and outcomes. Endocrine. 2012;42(3):471-482. doi:10.1007/s12020-012-9744-6

66. Stark Casagrande S, Fradkin JE, Saydah SH, Rust KF, Cowie CC. The prevalence of meeting $\mathrm{A} 1 \mathrm{C}$, blood pressure, and $\mathrm{LDL}$ goals among people with diabetes, 1988-2010. Diabetes Care. 2013;36 (8):2271-2279. doi: $10.2337 / \mathrm{dc} 12-2258$

67. Yu M, Mody R, Lando LF, et al. Characteristics associated with the choice of first injectable therapy among US patients with type 2 diabetes. Clin Ther. 2017;39(12):2399-2408. doi:10.1016/j.clinthera.2017.11.001

68. Elhussein A, Bancks M, Knowler WC, et al. 37-OR: racial and socioeconomic disparities in the use of newer classes of diabetes medications. Diabetes. 2020;69:37-OR. doi:10.2337/db20-37-OR

69. Toulis KA, Hanif W, Saravanan P, et al. All-cause mortality in patients with diabetes under glucagon-like peptide-1 agonists: a population-based, open cohort study. Diabetes Metab. 2017;43 (3):211-216. doi:10.1016/j.diabet.2017.02.003

70. Toulis KA, Willis $\mathrm{BH}$, Marshall $\mathrm{T}$, et al. All-cause mortality in patients with diabetes under treatment with dapagliflozin: a population-based, open-cohort study in the health improvement network database. J Clin Endocrinol Metab. 2017;102(5):1719-1725. doi:10.1210/jc.2016-3446

71. Svanstrom H, Ueda P, Melbye M, et al. Use of liraglutide and risk of major cardiovascular events: a register-based cohort study in Denmark and Sweden. Lancet Diabetes Endocrinol. 2019;7 (2):106-114. doi:10.1016/S2213-8587(18)30320-6

72. Longato E, Di Camillo B, Sparacino G, Tramontan L, Avogaro A, Fadini GP. Better cardiovascular outcomes of type 2 diabetic patients treated with GLP-1 receptor agonists versus DPP-4 inhibitors in clinical practice. Cardiovasc Diabetol. 2020;19(1):74.

73. Yang CT, Yang CY, Ou HT, Kuo S. Comparative cardiovascular safety of GLP-1 receptor agonists versus other glucose-lowering agents in real-world patients with type 2 diabetes: a nationwide population-based cohort study. Cardiovasc Diabetol. 2020;19(1):83. doi:10.1186/s12933-020-01053-0 
74. Pasternak B, Wintzell V, Eliasson B, et al. Use of glucagon-like peptide 1 receptor agonists and risk of serious renal events: Scandinavian cohort study. Diabetes Care. 2020;43(6):1326-1335. doi:10.2337/dc19-2088

75. Tonneijck L, van Raalte DH, Muskiet MHA. Liraglutide and renal outcomes in type 2 diabetes. $N$ Engl J Med. 2017;377(22):2195.

76. Gerstein HC, Colhoun HM, Dagenais GR, et al. Dulaglutide and renal outcomes in type 2 diabetes: an exploratory analysis of the REWIND randomised, placebo-controlled trial. Lancet. 2019;394 (10193):131-138.

77. Longato E, Di Camillo B, Sparacino G, Gubian L, Avogaro A, Fadini GP. Cardiovascular outcomes of type 2 diabetic patients treated with SGLT-2 inhibitors versus GLP-1 receptor agonists in real-life. BMJ Open Diabetes Res Care. 2020;8(1):e001451. doi:10. 1136/bmjdrc-2020-001451

78. Patorno E, Patrick AR, Garry EM, et al. Observational studies of the association between glucose-lowering medications and cardiovascular outcomes: addressing methodological limitations. Diabetologia. 2014;57(11):2237-2250. doi:10.1007/s00125-014-3364-z
79. Gokhale M, Sturmer T, Buse JB. Real-world evidence: the devil is in the detail. Diabetologia. 2020:1-12.

80. Liu Y, De A. Multiple imputation by fully conditional specification for dealing with missing data in a large epidemiologic study. Int J Stats Med Res. 2015;4(3):287-295. doi:10.6000/1929-6029. 2015.04.03.7

81. Sterne JA, Hernan MA, Reeves BC, et al. ROBINS-I: a tool for assessing risk of bias in non-randomised studies of interventions. BMJ. 2016;355:i4919. doi:10.1136/bmj.i4919

82. McAdam-Marx C, Nguyen H, Schauerhamer MB, et al. Glycemic control and weight outcomes for exenatide once weekly versus liraglutide in patients with type 2 diabetes: a 1-year retrospective cohort analysis. Clin Ther. 2016;38(12):2642-2651. doi:10.1016/j.clinthera. 2016.11.003

\section{Publish your work in this journal}

Diabetes, Metabolic Syndrome and Obesity: Targets and Therapy is an international, peer-reviewed open-access journal committed to the rapid publication of the latest laboratory and clinical findings in the fields of diabetes, metabolic syndrome and obesity research. Original research, review, case reports, hypothesis formation, expert opinion and commentaries are all considered for publication. The manuscript management system is completely online and includes a very quick and fair peer-review system, which is all easy to use. Visit http://www.dovepress.com/testimonials.php to read real quotes from published authors. 